\title{
Low cost plastic optical fiber sensor based on surface plasmon resonance
}

\author{
Víctor M. Muñoz-Berti*a,b, Ana C. López-Pérez ${ }^{\mathrm{a}, \mathrm{b}}$, Benito Alén ${ }^{\mathrm{a}}$, José Luis Costa-Krämer ${ }^{\mathrm{a}}$, Antonio \\ García-Martín $^{\mathrm{a}}$, Mauro Lomer ${ }^{\mathrm{b}}$, José Miguel López-Higuera ${ }^{\mathrm{b}}$ \\ anstituto de Microelectrónica de Madrid, IMM (CNM-CSIC), Isaac Newton 8 (PTM), 28760 Tres \\ Cantos (Madrid), Spain; \\ ${ }^{b}$ Photonics Engineering Group, University of Cantabria, ETSIIT, Avda. Los Castros s/n, 39005 \\ Santander, Spain.
}

\begin{abstract}
Surface plasmon excitation using a variation of Kretschmann method based on light guiding through an optical fiber has been extensively studied in the literature. But, due to its particularly bad propagation conditions, plastic optical fiber was not taken into account in documented experiments. We propose a low cost sensor using this type of fiber, in which we try to avoid the problems both through careful design and signal processing. First of all we discuss the sample fabrication and measurement in section 2; then the results obtained are discussed in section 3, including the problems faced because of the multimode character of the fiber, for which we propose alternative sample shapes as a mean of reducing them. As a conclusion we propose a roadmap to design a low cost sensor based in the structures studied in this paper.
\end{abstract}

Keywords: Plasmonics, plastic optical fiber, low cost sensor.

\section{INTRODUCTION}

The surface plasmon resonance (SPR) phenomenon is known from late 60's, since then this method has made great advances in manufacturing and applications. Sensors based on SPR had been developed, and efforts were being made to improve their performances. After the Kretschmann configuration for SPR excitation on a metal/dielectric interface using a prism ${ }^{1}$ numerous configurations based on integrated optics waveguides and optical fibers ${ }^{2,3}$ have been proposed. Introduction of optical fibers in the SPR sensing system is based on the guidance of light into the optical fiber by total internal reflection (TIR) where the core of an optical fiber replaces the prism in Kretschmann configuration. The basic principle involved in these sensors is the excitation of the surface plasmon existing on the air-gold interface through the coupling of light to the structure; this coupling is specific to a particular wavelength and sensitive to the refractive index, a dependence which allows its usage as a sensor. The advantages introduced by the optical fiber over prisms are their flexibility, small dimensions, easy handling and remote sensing capability. The metal film deposition on the core is made possible by the modification of the geometry of the fiber and the removal of a portion of the cladding ${ }^{4}$.

In this paper we study the excitation of surface plasmon resonance using a multimode plastic optical fiber, bent forming a U-shape and side-polished. The metal deposition is made on the polished surface in contact with the core. This configuration is intended to increase the sensitivity of the sensor response because when the radius of curvature decreases the sensitivity increases on the curved region, as is suggested by papers from other fields ${ }^{5}$. The proposed solution is advantageous in the manufacturing of this sensor in comparison to previous sensors. Much has been done in this field using single-mode fiber, but we study the behavior of light coupling inside a plastic optical fiber, and the possibility of taking advantage of the many modes propagated to excite surface plasmons at multiple wavelengths. Apart from the possibility of multiple resonances, plastic optical fiber is a low cost solution, so we can build low cost, singleuse sensors. First we propose a U-shaped geometry, based on the idea of radiating modes to the cladding so that they can interact with the metallic layer; then an alternative shape is proposed in order to avoid the difficulties imposed by the first configuration while still maximizing the coupling of light into the structure. After discussing the various geometries used to build the sensor we propose a straightforward explanation of the resulting curves, for finally discussing the problems faced when designing and testing this first configuration, which will be used as a basis for future work.

*victor@imm.cnm.csic.es

Fourth European Workshop on Optical Fibre Sensors, edited by José Luís Santos, Brian Culshaw, José Miguel López-Higuera, William N. MacPherson, Proc. of SPIE Vol. 7653, 765327

(c) 2010 SPIE · CCC code: $0277-786 X / 10 / \$ 18 \cdot$ doi: $10.1117 / 12.866537$

Proc. of SPIE Vol. $7653765327-1$ 


\section{EXPERIMENTAL RESULTS}

\subsection{Sample fabrication}

The sample consisted on a plastic optical $(980 \mu \mathrm{m}$ core diameter and $1000 \mu \mathrm{m}$ cladding diameter) fiber embedded in a PMMA block, with a double purpose: maintaining a constant curvature and easing the polishing process. The polishing process was carried out with a $3 \mu \mathrm{m}$ polishing paper in order to remove the plastic cover and part of the cladding. After this, in order to completely expose the core, we used a $0.9 \mu \mathrm{m}$ polishing paper.

In this experiment we used two types of samples, depending on the position of the gold deposition and labeled type a and type b, respectively. They are shown in Figure 1.
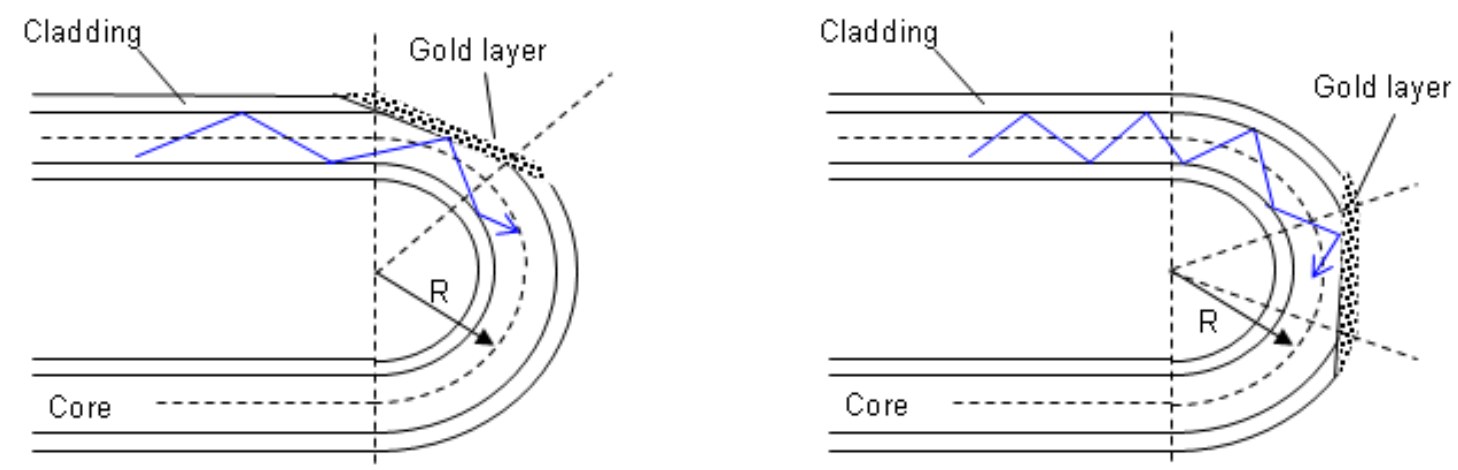

Figure 1 The two configurations tested in the current paper: type $a$, in which the gold layer appears at the beginning of the curve, where modes begin to be radiated to the cladding due to the curvature; type $b$, there the gold layer appears on the maximum curvature region.

The gold layer was deposited on the polished surface using the technique known as e-beam evaporation in high vacuum conditions. In the current samples a gold layer of $20 \mathrm{~nm}$ (sufficiently thin to allow for the excitation of a surface plasmon) was deposited at a rate of $0.6 \AA / \mathrm{s}$. This rate was chosen in order to preserve the surface of the plastic optical fiber, as a higher one could lead to the modification of its roughness, which could lead, as will be discussed later, to the alteration of the transmission spectrum.

\subsection{Sample measurement}

After the gold nanolayer had been deposited we measured the transmission spectrum. To do so, the light from a quartzhalogen lamp was coupled to one end of the POF and collected with a microscope objective through the other end. The transmitted light spectrum was recorded averaging 10 expositions of 1 second with an Ocean Optics USB4000 multichannel mini-spectrograph and correcting for the detector dark current. A first measurement of the fiber without the gold deposited layer was used as a reference, so that the resonant wavelengths became apparent when substracting the two signals: the spectrum with the gold layer and without it. The result is shown in Figure 2. As we can see there are multiple peaks, a result we shall discuss later. During the post processing of the measured data, we applied an additional average filter, to obtain smoother curves. Another sample configuration could result in cleaner signals, so this processing would not be necessary, as was confirmated by further measurements with planar samples (with a high curvature radius).

\section{DISCUSSION}

A plastic optic fiber is a highly multimode light guiding device, in which the nanolayer is illuminated by number of modes, which implies it can support multiple resonant wavelengths. The idea behind this conclusion is simple: each mode supports a different propagation constant, $\mathbf{k}$, or, from a geometrical point of view, a different incident angle. Localized surface plasmons vary their resonant wavelength with the incident angle, so, if we are exciting with a number of angles, it is possible to view the result shown in Figure 4 as the joint excitation of all of them, each one with its particular propagation constant and, thus, resonant wavelength. Whether we can take advantage of this multi-resonance in the sensing area or not is a topic we are currently studying. The difference between the sample before and after deposition is only the gold nanolayer, as the curvature was fixed by the PMMA block, so both theory and experiment 
suggest that the resonances are due to a surface plasmon excitation, with the optic fiber acting like the prism on Kretschmann's configuration. Anyway, a variation of the resonant wavelengths with the refractive index would be a definite proof of the presence of a surface plasmon.
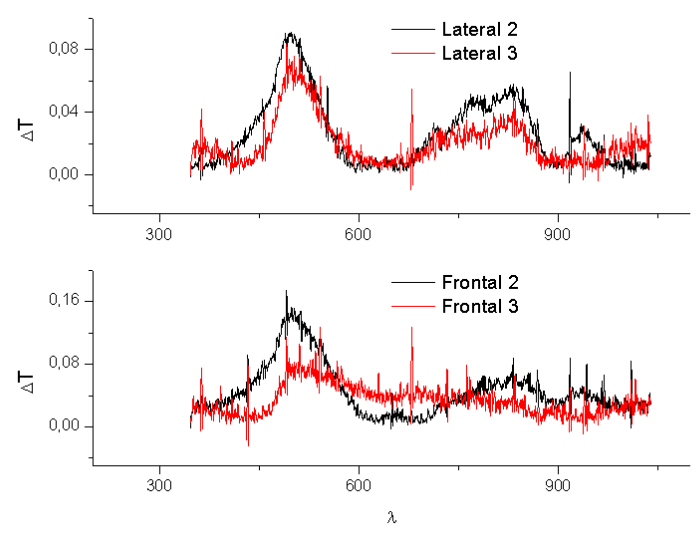

Figure 2 Experimental results of the first set of samples. The first curves show the results from type $a$ samples, while the second plot shows the results from type $b$. Both plots show the positive transmission difference between the samples before and after deposition.

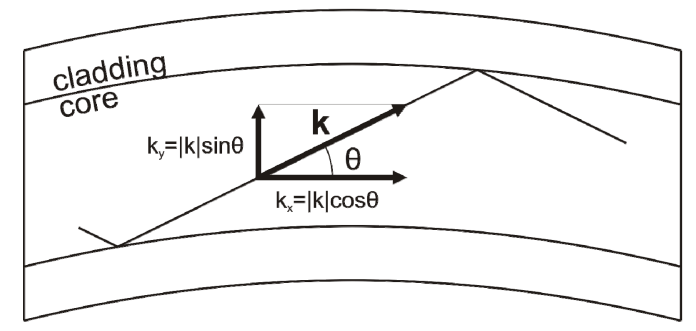

Figure 3 Orthogonal decomposition of the wavevector, k; relation between both components and the incident angle becomes evident.

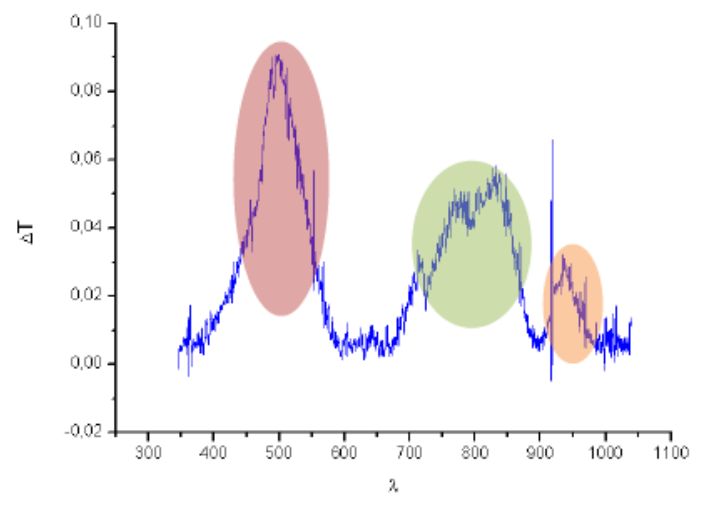

Figure 4 Detail of one of the resulting curves; observed resonances appear highlighted.

The simulation results (based on transfer matrix formalism ${ }^{6,7}$-not shown) also reveal that the layer thickness of the current samples $(20 \mathrm{~nm})$, although valid for our purpose, cab be varied to favor a better coupling, and, thus, greater signal to noise ratio. So, the next samples will be a test bench where we will try the proposed ways of improving the signal quality: sample shape and better plasmon excitation. New measurements carried out with different geometries and gold thicknesses initially prove our hypothesis, and show that changing the sample shape we can control the number of resonances. This result explains why type $a$ and type $b$ samples exhibit different peak intensity, as the number of radiated 
modes is higher in the latter. Those samples also demonstrated that the deposition rate did not affect the properties of the surfaced of the exposed fiber core, as the resulting spectrum after removing the deposited gold layer are the same as before the deposition ${ }^{8}$.

\section{CONCLUSIONS}

The noise present in our measurement we conducted is a direct result of the curvature of the sample, which has been reduced in subsequent experiments, as shown in Figure 5. Another pitfall of a curved sample is that an alteration of the superficial properties of the exposed core during the gold deposition process could lead to mode leakage, so that we could have lossy wavelengths not due to surface plasmon excitation, which could lead to two undesirable situations: additional lossy wavelengths and, in the worst case, in which all the peaks are due to this effect, the sensor could lose all its functionality. This mayor drawback of the plastic fiber based sensor is probably the most dangerous one, and great care is needed in the fabrication and characterization process.

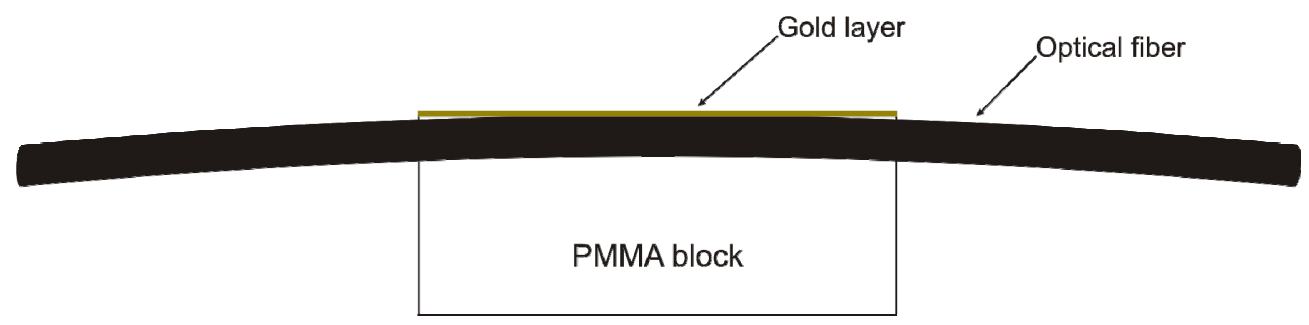

Figure 5 Shape of the new samples; they are almost planar (the curvature radius is very high) in order to avoid massive mode leakage and improve the quality of the transmitted signal.

Further measurements will require the addition of adhesive layers to ensure the adherence of the gold layer in the presence of liquid analyte, to provide the sufficient refractive index change to prove the sample function as a sensor and study its performance, and, through both simulation and experiment, optimize the sensor characteristics. Although such devices have been demonstrated through the literature, none of the authors have been aware of the potential of plastic optic fiber as a cheap means of building sensing apparatus, if they are carefully designed and fabricated; it is precisely this void the one we are trying to fill with our current research.

\section{ACKNOWLEDGMENTS}

While working on this project V. M. Muñoz-Berti and A. C. López-Pérez were supported through a CSIC "JAE Introddución a la investigación" grant. We would also like to thank Juan B. González-Díaz, from the Instituto de Microelectrónica de Madrid, for useful discussions and help with the simulations.

\section{REFERENCES}

[1] Kretschmann, E., "The determination of the optical constants of metals by excitation of surface plasmons", Zeitschrift fur Physik A, 241, 313 (1971).

[2] Villuendas, F. and Pelayo, J., "Optical fibre device for chemical sensing based on surface plasmon excitation", Sensors and Actuators A, 21, 142 (1990).

[3] Jorgenson, R. C. and Yee, S. S., "A fiber-optic chemical sensor based on surface plasmon resonance", Sensors and Actuators B, 8, 213 (1993).

[4] Sharma, A. K., Jha, R. and Gupta, B. D., "Fiber-optic sensors based on surface plasmon resonance: A comprehensive review", IEEE Sensors Journal, 7, 1118 (2007).

[5] Lomer, M., Lopez-Higuera J.M., et al., "Refractometric sensor based on induced losses in the region of transition from a curved side-polished POF fiber", OFS-19, Perth Australia, paper 7004-242 (2008).

[6] Kanso, M., Cuenot, S., Louarn, G., "Sensitivity of optical fiber sensor based on surface plasmon resonance: modelling and experiments", Plasmonics, 3, 49 (2008).

[7] Muñoz-Berti, V. M., García-Martín, A., Costa-Krämer, J. L., et al, to be published.

[8] Muñoz-Berti, V. M., López-Pérez, A. C., Alén, B., Costa-Krämer, J. L., García-Martín, A., Lomer, M., LópezHiguera, J.M., to be published. 